\title{
ON THE QUANTIZATION OF QUADRATIC MOMENTA
}

\author{
IZU VAISMAN
}

(Received 3 April 1981)

\begin{abstract}
Using geometric quantization, and accepting the quantum Hamiltonian of previous authors, we propose some candidate formulae for the quantum operator of an observable which is a quadratic form in the momenta.
\end{abstract}

\section{Introduction}

In this note, we undertake a partial mathematical discussion of the following quantization problem, formulated in the language of geometric quantization $[4,5$, $6,7,9]$.

Let us assume that we have a dynamical system whose configuration space is the orientable differentiable manifold $M, \operatorname{dim} M=n$, and whose phase space is the corresponding cotangent bundle $T^{*} M$. We shall denote by $\vartheta$ the canonical 1 -form on $T^{*} M$ and set $\Theta=-(1 /(2 \pi \hbar)) d \vartheta$, where $h=h / 2 \pi$, and $h$ is Planck's constant.

Let us also assume that the system has an associated electromagnetic field defined by a closed 2 -form $F$ on $M$, and that it has an electric charge $e$. Finally, the system will be assumed to have an energy defined by

$$
H=\frac{1}{2} g(u, u),
$$

where $u \in T^{*} M$, and $g$ is the contravariant tensor of a Riemannian metric on $M$, which will also be denoted by $g$.

(C) Copyright Australian Mathematical Society 1982 
Such a system has a basic symplectic form defined on $T^{*} M$ by

$$
\Omega=\Theta+e F,
$$

where, by an abuse of notation, we denote by $F$ both the considered form on $M$ and its lift to $T^{*} M$.

The quantization of a system of this type using geometric quantization has been discussed by J. Śniatycki in [5, 6] (see also Woodhouse [9]), where a formula is given for the corresponding quantum operator $\hat{H}$. This formula generalizes the formula of de Witt [3] and Cheng [2], who studied, by other methods, the case $F=0$.

Concerning other observables of the system, one knows, by the general theory, how to quantize the so-called configuration and momentum observables $[1,5]$, and the corresponding formulae are provided by Dirac's condition of the "preservation" of the Poisson brackets. But there is no well defined method for the quantization of the other observables, and it is even known that it is impossible to have a "good" quantization for all the observables of the system [1]. Consequently, it is usual to give quantization formulae for various observables, by more or less heuristic arguments, while the preservation of the brackets is no more always observed.

As a matter of fact, this remark also applies to the quantization of $H$. However, since the different methods of $[2,3,5,6]$ give the same result, we shall accept the formula for $\hat{H}$ in $[5,6]$, as a basic formula valid for the considered system.

Now, the problem which we have in mind is that of quantizing in the given system those observables which are of the form

$$
\gamma=\frac{1}{2} \gamma(u, u)
$$

where $u \in T^{*} M$, and $\gamma$ is an arbitrary second order contravariant symmetric tensor of $M$. We shall call such observables quadratic momenta.

Here also we shall be obliged to use some heuristic arguments, and the basic one will be that quantization of the other observables is determined by the classical energy $H$, and by its quantum operator $\hat{H}$, because of the fact that $H$ and $\hat{H}$ are fundamental from the physical viewpoint. This means that we shall be looking for quantum operators $\hat{\gamma}$, which are compatible with the known formula for $\hat{H}$. Another principle will be that of "preserving" as many Poisson brackets as possible.

By applying these principles, we shall arrive at some formulae for the operators $\hat{\gamma}$, which can be considered as mathematical candidates for the corresponding real-physical quantum operators. It is an interesting fact that these formulae will include the Riemannian metric $g$, and its curvature.

Finally, it should be emphasized that any discussion of whether the candidate formulae mentioned above are physically correct is beyond the scope of this note. 


\section{Previous results}

Let us start by a survey of the known results (see, for example, [6, 7]). First, we should assume that the following integrality condition is satisfied: $e F$ represents an integral cohomology class of $M$. This condition ensures the existence of a complex line bundle $K^{\prime}$ on $M$, endowed with a Hamiltonian metric $h^{\prime}$, and with a Hermitian connection $\nabla^{\prime}$ of curvature $2 \pi i e F$ where $i=\sqrt{-1}$. Namely, $K^{\prime}$ is defined by some local trivialization with local bases $\left\{\sigma^{\prime}\right\}$ such that

$$
h^{\prime}\left(\sigma^{\prime}, \sigma^{\prime}\right)=1 \quad \text { and } \quad \nabla_{X}^{\prime} \sigma^{\prime}=\beta^{\prime}(X) \sigma^{\prime},
$$

where

$$
\beta^{\prime}=(i / \hbar) e \alpha \quad \text { and } \quad F=(1 /(2 \pi \hbar)) d \alpha .
$$

(The second relation (2.2) defines the local 1-forms $\alpha$ since $F$ is closed).

Then, the lift $K$ of $K^{\prime}$ to $T^{*} M$ has some corresponding local bases $\{\sigma\}$ and it can be given a Hermitian metric $\chi$, and a Hermitian connection $\nabla$ by

$$
\chi(\sigma, \sigma)=1 \quad \text { and } \quad \nabla_{Z} \sigma=\beta(Z) \sigma,
$$

where

$$
\beta=(i / \hbar)(-\vartheta+e \alpha) .
$$

The curvature of the connection $\nabla$ is

$$
d \beta=2 \pi i \Omega,
$$

whence it follows that $K$ is the Kostant-Souriau line bundle of the dynamical system considered $[4,6,7,9]$.

On the other hand, $T^{*} M$ has the natural vertical polarization $S$ defined by the tangent spaces of the fibres. The natural atlas $\left(q^{a}, p_{a}\right)$ of $T^{*} M$, where $q^{a}$ $(a=1, \ldots, n)$ are local coordinates of a positively oriented atlas on $M$, and $p_{a}$ $(a=1, \ldots, n)$ are the natural covector coordinates with respect to the same atlas, is an adapted altas of $S$ [7]. Moreover, equation (2.4) shows that $\{\sigma\}$ is a corresponding distinguished basis of $K$ [7].

Furthermore, a metalinear structure of $\left(T^{*} M, S\right)$ can be fixed by choosing $\left[\operatorname{det}\left(\partial \tilde{q}^{a} / \partial q^{b}\right)\right]^{1 / 2}>0$ for every coordinate transformation $\tilde{q}(q)$ of the above mentioned positive atlas [7]. It follows that the line bundle $L$ whose cross-sections are half-forms on $T^{*} M$ is trivial. Indeed, let us consider the Riemannian metric $g$ of (1.1), and set $G=\operatorname{det} g$. Then the local functions $G^{1 / 4}$ lifted to $T^{*} M$ define an adapted half-form $\mathcal{G}$ which never vanishes.

Consequently, $K \otimes L \approx K$, and the wave functions are cross-sections of the form

$$
\Phi=\varphi \mathcal{S}
$$

where $\varphi$ is an adapted cross-section of $K[7]$. 
At this point, we can use the classical formulae of geometric quantization in order to obtain the quantum operators of configuration and momentum observables.

A configuration observable [1] is the lift of a function $f: M \rightarrow R$ to $T^{*} M$. Hence, it is adapted [7], and we have its quantum operator $\hat{f}$ given by

$$
\hat{f}(\Phi)=f \Phi \text {. }
$$

A momentum observable [1] is one of the form $\xi=u(X)$, where $u \in T^{*} M$, and $X$ is a fixed vector field on $M$. By means of the coordinates $\left(q^{a}, p_{a}\right)$ we have

$$
\xi=u(X)=\xi^{a} p_{a}
$$

(the Einstein summation convention being used throughout). The corresponding symplectic gradient [7] is

$$
\operatorname{sg} \xi=2 \pi \hbar\left(\xi^{a} \frac{\partial}{\partial q^{a}}+\eta_{a} \frac{\partial}{\partial p_{a}}\right)
$$

where the $\eta_{a}$ are some unimportant coefficients, and, by means of the usual quantization formulas [7] one obtains the quantum operator [1,5],

$$
\hat{\xi}(\Phi)=-i \hbar\left[\nabla_{X}^{\prime} \varphi+\frac{1}{2}(\operatorname{div} X) \varphi\right] \mathcal{G} .
$$

In (2.10), by an abuse of notation, we denoted by the same $\varphi$ the cross-section of $K^{\prime}$ induced by the adapted section $\varphi$ of $K$, while $\operatorname{div} X$ is computed by means of the metric $g$.

Let us also note that the operators $\hat{f}, \hat{\xi}$ satisfy commutation relations of the form

$$
\hat{A} \hat{B}-\hat{B} \hat{A}=\frac{1}{2 \pi i}\{A, B\}_{\Omega},
$$

where $\{A, B\}_{\Omega}$ is the Poisson bracket of the observables $A, B$ with respect to the symplectic form $\Omega$. Equation (2.11) is the correct technical meaning of Dirac's preservation of brackets condition mentioned in the Introduction. If $F=0,(2.11)$ becomes

$$
\hat{A} \hat{B}-\hat{B} \hat{A}=-i \hbar\{A, B\},
$$

where $\{A, B\}$ is the classical Poisson bracket.

Finally, the quantization of the energy $H$ of $(1.1)$, as given in $[2,3,5,6,9]$, is

$$
\hat{H}(\Phi)=-\frac{\hbar^{2}}{2}\left(g^{a b} \varphi_{/ a b}-\frac{1}{6} R \varphi\right) \mathcal{G},
$$

where $R$ is the scalar curvature of the metric $g$, and $\varphi_{/ a b}$ is a mixed covariant derivative defined as follows. Let $\varphi$ be a cross-section of the line bundle $K^{\prime}$. Then $\nabla^{\prime} \varphi$ is a $K^{\prime}$-valued l-form with the local components $\nabla_{\partial / \partial q^{a}}^{\prime} \varphi=\varphi_{/ a}$ (notation!), 
and, if $\Gamma_{a b}^{c}$ are the Christoffel symbols of the Riemannian metric $g$, we shall define

$$
\varphi_{/ a b}=\nabla_{\partial / \partial q^{b}}^{\prime} \varphi_{/ a}-\Gamma_{a b}^{c} \varphi_{/ c},
$$

the latter being obviously a $K^{\prime}$-valued tensor. Note that we shall also use a bar to denote the usual covariant derivatives with respect to $\Gamma_{a b}^{c}$ when required.

We have already mentioned in the Introduction that (2.13) cannot be deduced as straightforwardly as (2.7) and (2.10). Its deduction requires for instance the technical procedure known as $B K S$-pairing, for which we refer the reader to $[4,6$, 9]. Here, we shall accept without further proofs that equation (2.13) holds good.

\section{Quadratic momenta}

Now, we can proceed with the discussion of the quantization of observables of the form (1.3), whose coordinate expressions with respect to the atlas of Section 2 are

$$
\gamma=\frac{1}{2} \gamma^{a b} p_{a} p_{b} .
$$

For this reason, we call them quadratic momenta. Of course, we could try the $B K S$ pairing method, but it will be simpler to proceed in a more straightforward manner.

Let us begin by looking for the quantum operator attached to the observable $\xi^{2}$, where $\xi$ is given by (2.8). With the notation in Section 2, we get

$$
\{\xi, f\}_{\Omega}=2 \pi \hbar X f \text { and }\left\{\xi^{2}, f\right\}_{\Omega}=4 \pi \hbar \xi(X f),
$$

for any function $f$ on $M$, and the first relation (3.2) yields by means of (2.11)

$$
\hat{\xi}(f \Phi)-f \hat{\xi}(\Phi)=-i \hbar(X f) \Phi .
$$

On the other hand, (2.10) implies

$$
\widehat{f \xi}(\Phi)=f \hat{\xi}(\Phi)-\frac{1}{2} i \hbar(X f) \Phi .
$$

Hence, the second relation (3.2) leads to the following condition, which we consider as a necessary one since we have to "preserve as many brackets as possible":

$$
\widehat{\xi^{2}}(f \Phi)-f\left(\widehat{\xi^{2}}(\Phi)\right)=-2 i \hbar^{2}(X f) \hat{\xi}(\Phi)-\hbar(X X f) \Phi .
$$

Now, a simple computation based on (3.3) shows that, if we replace here $\widehat{\xi^{2}}$ by $\hat{\xi} \circ \hat{\xi}=\hat{\xi}^{2}$, the relation (3.5) will be identically verified. Hence, if we denote

$$
\widehat{\xi^{2}}-\hat{\xi}^{2}=\hbar^{2} A_{X},
$$

we get

$$
A_{X}(f \Phi)=f A_{X}(\Phi)
$$


which means that $A_{X}$ is a cross-section of $\operatorname{Hom}(K, K)$ and, locally, it consists of multiplication by a function.

Thereby, $\widehat{\xi^{2}}$ is defined up to a zero order operator, and, by means of (3.6) and (2.10), we get

$$
\begin{aligned}
\xi^{2}(\Phi)= & -\hbar^{2}\left\{\nabla_{X}^{\prime} \nabla_{X}^{\prime} \varphi+(\operatorname{div} X)\left(\nabla_{X}^{\prime} \varphi\right)+\frac{1}{2}(X \operatorname{div} X) \varphi+\frac{1}{4}(\operatorname{div} X)^{2} \varphi\right\} \mathcal{G} \\
& +\hbar^{2} A_{X}(\Phi),
\end{aligned}
$$

where $\Phi$ is given by (2.6).

Now let us consider $\gamma$ of (3.1). Here, we shall use another heuristic principle namely, the quantum operators should have a local character, that is, they should be compatible with the restriction to any open neighbourhood. This is usually admitted in quantization. Accordingly, let us assume that our computations are done over a neighbourhood $U$ which is small enough and on which rank $\gamma=s$. Then we can set

$$
\gamma_{/ U}=\frac{1}{2} \sum_{k=1}^{s} \varepsilon_{k} \xi_{k}^{2}
$$

where $\xi_{k}=u\left(v_{k}\right),\left(u \in T^{*} M\right)$, for some vector fields $v_{k}$ defined on $U$, and $\varepsilon_{k}= \pm$. Componentwise (3.9) means

$$
\gamma^{a b}=\sum_{k=1}^{s} \varepsilon_{k} v_{k}^{a} v_{k}^{b}
$$

Correspondingly, we can compute $\hat{\gamma}$ by means of (3.8) applied to the vector fields $v_{k}$. It is easy to show that

$$
\nabla_{v_{k}}^{\prime} \nabla_{v_{k}}^{\prime} \varphi+\left(\operatorname{div} v_{k}\right) \nabla_{v_{k}}^{\prime} \varphi=v_{k}^{a} v_{k}^{b} \varphi_{/ a b}+\left(v_{k}^{a} v_{k}^{b}\right)_{/ b} \varphi_{/ a},
$$

where $\varphi_{/ a b}$ is defined by (2.14), and (3.11) yields

$$
\sum_{k=1}^{s} \varepsilon_{k}\left[\nabla_{v_{k}}^{\prime} \nabla_{v_{k}}^{\prime} \varphi+\left(\operatorname{div} v_{k}\right) \nabla_{v_{k}}^{\prime} \varphi\right]=\gamma^{a b} \varphi_{/ a b}+\gamma_{/ b}^{a b} \varphi_{/ a} .
$$

On the other hand, we have

$$
v_{k}\left(\operatorname{div} v_{k}\right)+\frac{1}{2}\left(\operatorname{div} v_{k}\right)^{2}=v_{k}^{a} v_{k / b a}^{b}+\frac{1}{2} v_{k / a}^{a} v_{k / b}^{b} .
$$

In order to compute the contribution of these terms, we derive from (3.10)

$$
\gamma_{/ a b}^{a b}=\sum_{k=1}^{s} \varepsilon_{k}\left(v_{k / a b}^{a} v_{k}^{b}+v_{k / a}^{a} v_{k / b}^{b}+v_{k / b}^{a} v_{k / a}^{b}+v_{k}^{a} v_{k / a b}^{b}\right),
$$

and, next, use the classical formula

$$
v_{k / a b}^{b}-v_{k / b a}^{b}=R_{c a b}^{b} v_{k}^{c},
$$


where $R$ is the curvature tensor of the metric $g$. Then, (3.14) becomes

$$
\begin{aligned}
\gamma_{/ a b}^{a b} & =\sum_{k=1}^{s} \varepsilon_{k}\left(2 v_{k}^{a} v_{k / b a}^{b}+v_{k / a}^{a} v_{k / b}^{b}+v_{k / b}^{a} v_{k / a}^{b}+v_{k}^{a} v_{k}^{c} R_{c a b}^{b}\right) \\
& =2 \sum_{k=1}^{s} \varepsilon_{k}\left[v_{k}\left(\operatorname{div} v_{k}\right)+\frac{1}{2}\left(\operatorname{div} v_{k}\right)^{2}\right]+\sum_{k=1}^{s} \varepsilon_{k} v_{k / b}^{a} v_{k / a}^{b}+\gamma^{a c} R_{a c},
\end{aligned}
$$

where $R_{a c}$ is the Ricci curvature of the metric $g$.

From these results, we deduce that the quantum operator $\hat{\gamma}$ should be of the form

$$
\hat{\gamma}(\Phi)=-\frac{\hbar^{2}}{2}\left\{\gamma^{a b} \varphi_{/ a b}+\gamma_{/ a}^{a b} \varphi_{/ b}+\frac{1}{4}\left(\gamma_{/ a b}^{a b}-\gamma^{a b} R_{a b}\right) \varphi\right\} \mathcal{G}+\frac{\hbar^{2}}{2} B_{\gamma}(\Phi)
$$

where

$$
B_{\gamma}(\Phi)=\sum_{k=1}^{s} \varepsilon_{k}\left(A_{v_{k}}(\Phi)+\frac{1}{4} v_{k / b}^{a} v_{k / a}^{b} \Phi\right),
$$

which is an operator defined by a cross section of $\operatorname{Hom}(K, K)$.

In particular, (3.17) can be applied for $\gamma=H$ of (1.1), and this gives

$$
\hat{H}(\Phi)=-\frac{\hbar^{2}}{2}\left(g^{a b} \varphi_{/ a b}-\frac{1}{4} R \varphi\right) \mathcal{G}+\frac{\hbar^{2}}{2} B_{H}(\Phi),
$$

where, unless well chosen, $B_{H}$ will depend on the choice of the local bases $v_{k}$. This happens if, for instance, we make the mathematically natural choice $A_{X}=0$ for every $X$. Hence, as surprising as it might seem, we should consider $\widehat{\xi^{2}} \neq \hat{\xi}^{2}$, while the difference depends on the metric $g$.

Another mathematically natural choice is

$$
A_{X}(\Phi)=-\frac{1}{4} \xi_{/ b}^{a} \xi_{/ a}^{b} \Phi,
$$

which implies $B_{\gamma}=0$ for every $\gamma$, but then (3.19) does not reduce to the already accepted formula (2.13). (See, however, the remark of [8, page 28] about the possibility of having in (2.13) the coefficient $\frac{1}{4}$ instead of $\frac{1}{6}$, and the remark of [3, page 395] about the ambiguity of this coefficient.)

Now, since our principle has been to accept equation (2.13) as it stands, we must look for a corresponding choice of $A_{X}$ and $B_{\gamma}$. If we are taking a closer look at equations (2.13), and (3.17)-(3.19), we can see that the convenient choice is

$$
\begin{aligned}
A_{X}(\Phi) & =\frac{1}{6}\left\{X(\operatorname{div} X)+\frac{1}{2}(\operatorname{div} X)^{2}-\xi_{/ b}^{a} \xi_{/ a}^{b}\right\} \Phi \\
& =\left\{-\frac{1}{4} \xi_{/ b}^{a} \xi_{/ a}^{b}+\frac{1}{12}\left(\xi^{a} \xi^{b}\right)_{/ a b}-\frac{1}{12} R_{a b} \xi^{a} \xi^{b}\right\} \Phi,
\end{aligned}
$$

where the last equality follows by a computation similar to that of (3.16). 
With this choice, (3.19) reduces to (2.13), and our candidate for the quantum operator of a general quadratic momentum, as deduced from (3.17), will be

$$
\hat{\gamma}(\Phi)=-\frac{\hbar^{2}}{2}\left\{\gamma^{a b} \varphi_{/ a b}+\gamma_{/ a}^{a b} \varphi_{/ b}+\frac{1}{6}\left(\gamma_{/ a b}^{a b}-\gamma^{a b} R_{a b}\right) \varphi\right\} \mathcal{G} .
$$

We shall also have, in particular,

$$
\begin{aligned}
\widehat{\xi^{2}} \Phi=-\hbar^{2}\left\{\nabla^{\prime}{ }_{X} \nabla^{\prime}{ }_{X} \varphi+(\operatorname{div} X) \nabla^{\prime}{ }_{X} \varphi\right. & +\frac{1}{3}(X \operatorname{div} X) \varphi \\
& \left.+\frac{1}{6}(\operatorname{div} X)^{2} \varphi+\frac{1}{6}\|D \xi\|^{2} \varphi\right\} \mathcal{G},
\end{aligned}
$$

where $D \xi$ denotes the tensor $\xi_{/ b}^{a}$.

\section{Appendix}

In this appendix, we should like to call the attention of the reader to the possibility of using local coordinates on the phase space $T^{*} M$ such that $H$ assumes the canonical form.

Namely, it is known that local orthonormal bases consisting of local vector fields $v_{a}(a=1, \ldots, n)$ are available, and the vector bundle $T^{*} M$ has the local dual cobases $\omega^{a}$ defined by

$$
\omega^{a}\left(v_{b}\right)=\delta_{b}^{a} \quad(a, b=1, \ldots, n) .
$$

Then we can define on the manifold $T^{*} M$ the local coordinates $\left(q^{a}, s_{a}\right)$, where the $q^{a}$ are as in the foregoing sections, and the $s_{a}$ are the components of the covectors with respect to the cobases $\omega^{a}$ or, equivalently,

$$
s_{a}(u)=u\left(v_{a}\right) \text {. }
$$

The transition functions for these local coordinates are

$$
\tilde{q}^{a}=\tilde{q}^{a}\left(q^{b}\right), \quad \tilde{s}_{a}=\alpha_{a}^{b}\left(q^{c}\right) s_{b},
$$

where $a, b, c=1, \ldots, n$, and $\left(\alpha_{a}^{b}\right)$ is an orthogonal matrix.

Now the Hamiltonian $H$ becomes

$$
H=\frac{1}{2} \sum_{a=1}^{n} s_{a}^{2}
$$

and we shall say that $\left(q^{a}, s_{a}\right)$ are canonical coordinates with respect to $H$.

Obviously, the canonical 1-form $\vartheta$ on $T^{*} M$ becomes

$$
\vartheta=s_{a} \omega^{a} .
$$

In order to compute $d \vartheta$, let us introduce the local forms

$$
\omega_{a}^{b}=\gamma_{a c}^{b} \omega^{c} \quad\left(\gamma_{a c}^{b}+\gamma_{b c}^{a}=0\right)
$$


of the Levi-Civita connection of the metric $g$ defined by $H$, with respect to the basis $v_{a}$. As it is well known, we have then the structure equations

$$
d \omega^{a}=\omega^{b} \wedge \omega_{b}^{a},
$$

and we derive

$$
\Theta=-(1 /(2 \pi \hbar)) D s_{a} \wedge \omega^{a},
$$

where

$$
D s_{a}=d s_{a}-s_{b} \omega_{a}^{b} .
$$

Let us also note that the horizontal lifts of the vector fields $v_{a}$ to $T^{*} M$ are given by

$$
\nu_{a}=v_{a}^{b} \frac{\partial}{\partial q^{b}}+\gamma_{b a}^{c} s_{c} \frac{\partial}{\partial s_{b}} .
$$

Now the basic symplectic form (1.2) becomes

$$
\Omega=-(1 /(2 \pi \hbar)) D s_{a} \wedge \omega^{a}+e F,
$$

and one can proceed from here with the study of the quantization problem.

\section{References}

[1] R. Abraham and J. E. Marsden, Foundations of mechanics (Benjamin-Cummings Inc., Reading, Mass., 2nd Edition, 1978).

[2] K. S. Cheng, "Quantization of a general dynamical system by Feynman's path integration formulation", J. Math. Phys. 13(1972), 1723-1726.

[3] B. S. de Witt, "Dynamical theory in curved spaces I", Rev. Mod. Phys. 29(1957), 377-397.

[4] D. J. Simms and N. M. J. Woodhouse, "Lectures on geometric quantization", Lecture Notes in Physics 53 (Springer-Verlag, New York, 1976).

[5] J. Śniatycki, "Application of geometric quantization in quantum mechanics", Lecture Notes in Mathematics 676 (Springer-Verlag, New York, 1978), 357-368.

[6] J. Śniatycki, Geometric quantization and quantum mechanics (Springer-Verlag, Berlin, 1980).

[7] I. Vaisman, "A coordinatewise formulation of geometric quantization", Ann. Inst. H. Poincaré (A) 31 (1979), 5-24.

[8] A. Weinstein, “Quasi-classical mechanics on spheres", Symp. Math. 14 (1974), 25-32.

[9] N. M. J. Woodhouse, Geometric quantization (Clarendon Press, Oxford, 1980).

\section{Department of Mathematics \\ University of Haifa \\ Haifa \\ Israel}

